\title{
A Mathematical Model for Control and Elimination of the Transmission Dynamics of Measles
}

\author{
Stephen Edward, Kitengeso Raymond E., Kiria Gabriel T., Felician Nestory, Mwema Godfrey G., \\ Mafarasa Arbogast P.
}

Department of Mathematics, College of Natural and Mathematical Sciences, University of Dodoma (UDOM), Dodoma, Tanzania

\section{Email address:}

stephenmwaihuti@yahoo.com (S. Edward), temuray@yahoo.com (Kitengeso R. E.), gabrielkiria@yahoo.com (Kiria G. T.), nestorysamson@gmail.com (F. Nestory), godfreymwema@yahoo.com (Mwema G. G.), parbogast69@gmail.com (Mafarasa A. P.)

\section{To cite this article:}

Stephen Edward, Kitengeso Raymond E., Kiria Gabriel T., Felician Nestory, Mwema Godfrey G., Mafarasa Arbogast P.. A Mathematical Model for Control and Elimination of the Transmission Dynamics of Measles. Applied and Computational Mathematics.

Vol. 4, No. 6, 2015, pp. 396-408. doi: 10.11648/j.acm.20150406.12

\begin{abstract}
Despite the availability of measles vaccine since 1963, the infectious disease is still endemic in many parts of the world including developed nations. Elimination of measles requires maintaining the effective reproduction number less than unity, $R_{e}<1$ as well as achieving low levels of susceptibility. Infectious diseases are great field for mathematical modeling, and for connecting mathematical models to primary or secondary data. In this project, we concentrated on the mathematical model for control and elimination of transmission dynamics of measles. We have obtained disease free equilibrium (DFE) point, effective reproduction number and basic reproduction number for the model. Simulations of different variables of the model have been performed and sensitivity analysis of different embedded parameters has been done. MATLAB has been used in simulations of the ordinary differential equations (ODEs) as well as the reproduction numbers.
\end{abstract}

Keywords: Measles, Vaccination, Immunity, Mathematical Modelling, Herd Immunity

\section{General Introduction}

In this section we discussed general description of measles, statement of the problem, objectives of the project, project questions and significance of the project.

\subsection{General Description of Measles}

In this section we discussed background of measles, symptoms of measles, transmission of measles, treatment of measles, immunization of measles and the current situation of measles.

\subsubsection{Background of Measles}

Measles (also called rubeola) is a highly contagious viral infection that can be found around the world through person-to-person transmission mode, with over $90 \%$ attack rate among susceptible persons. It is the first worth eruptive fever occurring during childhood. The measles virus is a paramyxovirus, genus morbillivirus. Even though an effective vaccine is available and widely used, measles continues to occur even in developed countries. Children under five years are most at risk. Measles infects about 30 to 40 million children each year and causing mortality of over one million often from complication related to pneumonia, diarrhea and malnutrition [2]. One of the earliest written descriptions of measles as a disease was provided by an Arab physician in the $9^{\text {th }}$ century who described differences between measles and smallpox in his medical notes. A Scottish physician, Francis Home, demonstrated in 1757 that measles was caused by an infectious agent present in the blood of patients. In 1954 the virus that causes measles was isolated in Boston, Massachusetts, by John F. Enders and Thomas C. Peebles. Before measles vaccine, nearly all children got measles by the time they were 15 years of age [3].

\subsubsection{Symptoms of Measles}

The main symptoms of measles are fever, runny nose, cough and a rash all over the body, it also produces characteristics-red rash and can lead to serious and fatal complications including pneumonia, diarrhea and encephalitis. Many infected children subsequently suffer blindness, deafness or impaired vision. Measles confer lifelong immunity from further attacks [1].

\subsubsection{Transmission of Measles}

Measles is a highly contagious virus that lives in the nose and throat mucus of an infected person. It can spread to others 
through coughing and sneezing. Also, measles virus can live for up to two hours in an airspace where the infected person coughed or sneezed. If other people breathe the contaminated air or touch the infected surface, then touch their eyes, noses, or mouths, they can become infected. Measles is so contagious that if one person has it, $90 \%$ of the people close to that person who are not immune will also become infected. Infected people can spread measles to others from four days before through four days after the rash appears. Measles is a disease of humans; measles virus is not spread by any other animal species.

\subsubsection{Treatment of Measles}

There is no specific treatment for measles. People with measles need bed rest, fluids, and control of fever. Patients with complications may need treatment specific to their problem.

\subsubsection{Immunization of Measles}

There are two doses for measles vaccine, the first dose of Measles Mumps-Rubella (MMR) should be given on or after the child's first birthday; the recommended age range is from 12-15 months. A dose given before 12 months of age will not be counted, so the child's medical appointment should be scheduled with this in mind. The second dose is usually given when the child is 4-6 years old, or before he or she enters kindergarten or first grade. However, the second dose can be given earlier as long as there has been an interval of at least 28 days since the first dose. The first dose of MMR produces immunity to measles in $90 \%$ to $95 \%$ of recipients. The second dose of MMR is intended to produce immunity in those who did not respond to the first dose, but a very small percentage of people may not be protected even after a second dose. Anyone who had a severe allergic reaction (e.g., generalized hives, swelling of the lips, tongue, or throat, difficulty breathing) following the first dose of MMR should not receive a second dose. Anyone knowing they are allergic to an MMR component (e.g., gelatin, neomycin) should not receive this vaccine. As with all live virus vaccines, women known to be pregnant should not receive the MMR vaccine, and pregnancy should be avoided for four weeks following vaccination with MMR. Children and other household contacts of pregnant women should be vaccinated according to the recommended schedule. Women who are breastfeeding can be vaccinated. Severely immuno- compromised people should not be given MMR vaccine. This includes people with conditions such as congenital immunodeficiency, AIDS, leukemia, lymphoma, generalized malignancy, and those receiving treatment for cancer with drugs, radiation, or large doses of corticosteroids. Household contacts of immunocompromised people should be vaccinated according to the recommended schedule. Although people with AIDS or HIV infection with signs of serious immunosuppression should not be given MMR, people with HIV infection that do not have laboratory evidence of severe immunosuppression can and should be vaccinated against measles.

\subsubsection{Current Situation of the Disease}

Each year in the United States about 450-500 people died because of measles, 48,000 were hospitalized, 7,000 had seizures, and about 1,000 suffered permanent brain damage or deafness. Today there are only about 60 cases a year reported in the United States, and most of these originate outside the country. For 65 countries with adequate vital registration data $(\geq 85 \%$ of estimated deaths of children younger than 5 years registered and coded), they used the reported number of measles deaths. These deaths accounted for less than $0.01 \%$ of global measles mortality, according to vital registration data and estimated mortality [3]. For 128 remaining countries with inadequate vital Registration data, WHO estimated country-specific measles deaths through a three-step process. WHO estimated annual measles incidence on the basis of reported measles cases for each country, then WHO distributed estimated incidence across age groups, and finally WHO calculated the number of deaths in each age class by applying age-specific and country-specific measles Case-Fatality Ratios (CFRs). Measles cases and vaccination coverage are reported annually to WHO by all member states through the WHO/ UNICEF Joint Reporting Form [4]. WHO derived coverage estimates for the first routine dose of Measles-Containing Vaccine (MCV1) from reported coverage data and survey results by use of computational logic [5]. Measles cases reported through surveillance systems typically represent a fraction of the true number of cases because many children do not present for medical attention and when medical care is sought, cases can be misdiagnosed or not reported to central authorities [6].

\subsection{Statement of the Problem}

Despite the availability of the measles vaccine since 1963, the infectious disease is still endemic in many parts of the world including developed nations. The disease has continued causing both economic and health problems to large population worldwide mostly affecting children. Due to these impacts, this study aims to develop a mathematical model for control and elimination of the transmission dynamics of measles.

\subsection{Objectives of the Project}

\subsubsection{Main Objective of the Project}

The main objective of this project is to develop a mathematical model for control and elimination of the transmission dynamics of measles.

\subsubsection{Specific Objectives of the Project}

This project intends to achieve the following specific objectives:

i. Formulate a mathematical model for control and elimination of the transmission dynamics of measles.

ii. To obtain the disease free equilibrium (DFE) point.

iii. To obtain and analyze the effective reproduction number and basic reproduction number.

iv. To perform sensitivity analysis of each parameter involved in the model.

v. To perform simulation of the mathematical model. 


\subsection{Project Questions}

Important questions about control and elimination of the transmission dynamics of measles to be answered by this project are:

i. Can a mathematical model for control and elimination of the transmission dynamics of measles be formulated?

ii. Does the disease free equilibrium (DFE) point for the model exist?

iii. Do the effective reproduction number and basic reproduction number for the model exist?

iv. How sensitive is each embedded parameter?

v. Can measles be eliminated from a population?

\subsection{Significance of the Project}

The significances of this project are as follows:

i. The analysis of dynamics of measles transmission can be used to predict measles outbreak before it occurs.

ii. The government and health organizations can use findings of this project to plan vaccination programmes and hence prevent future measles outbreak.

iii. The public will participate in vaccination programmes because they will be aware of how it is best way to protect future measles outbreaks.

iv. This project will contribute to improve future studies of measles mathematical modeling.

v. Detailed explanation of transmission of measles between different groups in a population and sensitivity analysis of each parameter can help to control measles outbreak when it occurs.

\section{Literature Review}

In this section we review in brief mathematical models of measles developed previously. Mathematical models have a long history of been used by different goverments and organisations in the world in controlling and elimination strategies of infectious diseases. The following are some of mathematical models developed to study transimission dynamics of measles using different approaches:

[15] Developed a mathematical model for control of measles epidemiology. They used SEIR model to determine the impact of exposed individuals at latent period through the stability analysis and numerical simulation.

[16] Discussed modeling the effects of vaccination on the transmission dynamics of measles. In their study they divided the total population into five classes that is they used SEIR model and added the class of passively immune infants. In their study they tried to predict an optimal vaccine coverage level needed to control the spread of measles.

[10] Developed a mathematical model to the impact of the Measles Control Campaign (MCC) on the potential for measles transmission in Australia. They divided the population into five age groups and they used serosurvey results and vaccine coverage estimates to calculate the change in Basic Reproductive Number after measles control campaign (MCC). Their study realized that the Australian MCC had a significant impact on the transmission dynamics of measles and sustained efforts are required to improve coverage of two doses of MMR and to ensure elimination of indigenous measles transmission.

[7] Developed a mathematical model for measles epidemics in Ireland. The aim of her study was to establish a mathematical model for measles epidemics and to predict the levels of vaccination coverage required in Ireland in order to eradicate the disease.

[13] Studied the modeling and simulation of the dynamics of the transmission of measles. They used SEIR model to discuss dynamics of measles infection and address the stability and disease free and endemic equilibrium. The impact of vaccination in the control and elimination of measles was not discussed in their study.

[19] Based on the dynamical analysis of a new model for measles infection. His study used SEIR model modified by adding vaccinated compartment. His model determined the required vaccination coverage and dosage that will guarantee eradication of measles within a population.

[11] Performed a study in the modeling measles re-emergence as a result of waning of immunity in vaccinated populations. They developed an age structured mathematical model for measles transmission in vaccinated population. One of the principal insights gained from their model is that waning of immunity and subsequent mild subclinical infection in vaccines would not necessarily result in a rapid re-emergence of measles, but that the re-emergence is realistic and essentially depends on parameters for which no good estimates exist.

[17] Performed a study on mathematical modeling on the control of measles by vaccination. In their study SEIR model was used to show control of measles by vaccination. Their study recommended introduction of mass vaccination programme and improvement in early detection of measles cases to minimize transmission.

[9] Performed a study on predicting and preventing measles epidemics in New Zealand: application of mathematical model. In their study they used a deterministic SIR to model the dynamics of measles under varying immunization strategies in a population with size and age structure. The model successfully predicted an epidemic in 1997 and was instrumental in the decision to carry out an intensive MMR (measles, mumps and rubella) immunization campaign in that year in New Zealand.

[12] Developed a mathematical model for control of measles by vaccination. In their study they used SEIR model to study the population. Their results rely upon locally stability of the disease-free equilibrium point. They studied the local stability of endemic equilibrium by linearization, Jacobian matrix and Routh-Hurwitz theorem. These techniques were not suitable to know if the free-disease equilibrium point is globally stable; in such case, the disease can be eradicated irrespective the initial sizes of the compartment, as encountered in the real situation. Another limitation they realized is the lack of success when prospecting global stability for SEIR epidemiological models with non-constant population.

[18] Performed a mathematical model of measles with 
vaccination and two phases of infectiousness. They followed the SIR modeling approach hence they partitioned the total population is into Susceptible, Infectious and Recovered compartments. Their study realized that the disease will certainly be eliminated if all susceptible are vaccinated. Achieving a $100 \%$ vaccination coverage is impractical but if the goal is set to $100 \%$ then we just might hit the $\geq 94 \%$ vaccine coverage which is the herd immunity for measles. Since measles is predominantly found among children aged 5 years and below, they therefore suggested that the measles vaccine should be made compulsory such that no child is allowed to enter school without evidence of at least two dose measles vaccination.

[14] Developed a mathematical model for the study of measles in Cape Coast Metropolis. They used SEIR model to describe the transmission dynamics of measles. The model has shown success in attempting to predict the causes of measles transmission within a population. The model strongly indicated that the spread of a disease largely depend on the contact rates with infected individuals within a population. Their study also realized that if the proportion of the population that is immune exceeds the herd immunity level for the disease, then the disease can no longer persist in the population. Thus if this level can be exceeded by mass vaccination, then the disease can be under control. The model also pointed out that early detection has a positive impact on the reduction of measles transmission that is there is a need to detect new cases as early as possible so as to provide early treatment for the disease. More people should be educated in order to create awareness to the disease transmission so that society will be aware of this deadly disease.

[20] Performed a study on controlling measles using supplemental immunization activities: a mathematical model to inform optimal policy. They developed DynaMICE (Dynamic Measles Immunization Calculation Engine), an age-stratified model of measles infection transmission in vaccinated and unvaccinated individuals. The population in the model can be susceptible to measles, infected with measles or recovered from measles (and hence have lifelong immunity) that is SIR model. In their study, the rate at which infection occurs in the susceptible population depends on the existing proportion of the population that is already infected, as well as the effective contact rate between different age groups. Individuals age discretely, in one-year increments, at the end of each year [22], between 0 and 100 years old. Furthermore, both numerical simulation and mathematical analysis indicated that a single Supplemental Immunization Activity (SIA) will not control measles outbreaks in any of the countries with high burden of measles. However, regular Supplemental Immunization Activities (SIAs) at high coverage are able to control measles transmission, with the periodicity of SIA campaigns determined by population demo-graphics and existing MCV1 coverage.

\section{Model Formulation and Analysis}

\subsection{Description of the Compartmental Mathematical Model}

In this section a deterministic, compartmental mathematical model to describe the transmission dynamics of measles is formulated. It is assumed that the population is homogeneously mixing and reflects increasing dynamics such as birth and immigration, Per Capita birth rate is time constant, Per Capita natural mortality rate is time constant, individual can be infected through direct contact with an infectious individual, on recovery the individual obtains permanent infection-acquired immunity that is an individual cannot be infected again by measles and individual who has attended first and second dose of vaccine consecutively receive permanent immunity to measles.

The total population $(N)$ is divided into the following epidemiological classes: Susceptible, $S$ (Individuals who may get the disease); Exposed or Latent, $E$ (Individuals who are exposed to the disease); Infected, $I$ (Individuals who have the disease and are able to transfer it to others); Recovered, $R$ (Individuals who have permanent infection-acquired immunity and those who received the second dose of vaccine) and Vaccinated, $V$ (Individuals who have received first dose of vaccine). It is assumed that proportions $\phi$ of newborns and $\rho$ of immigrants receive first dose of vaccine and join the Vaccinated class, $V$ at rates $\pi$ and $\Lambda$ respectively. While the compliments $1-\phi$ and $1-\rho$ join the susceptible class, $S$ at the same rates. Susceptible individuals may be vaccinated at the rate $\varepsilon$ and join the Vaccinated class, $V$. If there is an adequate contact of a Susceptible individual with an Infective individual then transmission may occur, thus the susceptible individuals may join the Exposed class, $E$ at the rate $\lambda$. When Latent period ends, exposed individuals may progress to the Infectious class, $I$ at rate $\sigma$. After some treatment, infectious individuals may recover and join the recovery class, $R$ at rate $\eta$. Since the disease is fatal, infected individuals may die due to the disease at the rate $\delta$ or die naturally at rate $\mu$. The recovery class, $R$ consists of those with permanent infection-acquired immunity and those who received the second dose of vaccine, $\omega$. We assume that first vaccine does not confer lifelong immunity, it wanes with time at the rate $\alpha$, therefore a proportion $\theta$ of first dose vaccinated individuals who receive second vaccine may be conferred permanent immunity whereas the compliment $(1-\theta)$ who may skip second vaccination become susceptible to the disease at the rate $\alpha$. Basically our new model is an SVEIR model.

\subsection{Description of Variables and Parameters}

The following tables describe the variables and parameters used in this model:

Table 1. Variables used in the model.

\begin{tabular}{ll}
\hline Variable & Description \\
\hline$S$ & The number of Susceptible individuals at time $\mathrm{t}$ \\
$E$ & The number of Exposed individuals at time $\mathrm{t}$ \\
$I$ & The number of Infected individuals at time $\mathrm{t}$ \\
$R$ & The number of Recovered individuals at time $\mathrm{t}$ \\
$V$ & The number of Vaccinated individuals at time $\mathrm{t}$ \\
$N$ & The total population at time t \\
\hline
\end{tabular}


Table 2. Parameters used in the model .

\begin{tabular}{ll}
\hline Parameter & Description \\
\hline$\pi$ & Per Capita birth rate \\
$\phi$ & Constant Immigration rate \\
$\phi-\phi$ & Proportions of newborns who are vaccinated \\
$\rho$ & Proportion of newborns who are not vaccinated \\
$1-\rho$ & Proportions of immigrants who are vaccinated \\
$a$ & Proportion of immigrants who are not vaccinated \\
$c$ & Arrival rate \\
$\delta$ & Per Capita contact rate \\
$\mu$ & Death due to disease \\
$\beta$ & Per Capita natural mortality rate \\
$\lambda$ & Probability of one infected individual to become infectious \\
$\sigma$ & Force of infection, $\lambda=\frac{\beta c I}{N}$ \\
$\eta$ & Progression rate from latent to infectious \\
$\alpha$ & Recovery rate of treated infectious individuals \\
$\omega$ & The rate of waning of first dose of vaccine \\
$\varepsilon$ & The rate of receiving second dose of vaccine \\
$\theta$ & Proportion of individuals who received a first vaccination \\
$1-\theta$ & Proportion of individuals who are vaccinated twice \\
\hline & Proportion of individuals who are not vaccinated twice \\
\hline
\end{tabular}

\subsection{Compartmental Diagram}

The description of measles dynamics can be summarized by compartmental diagram below:

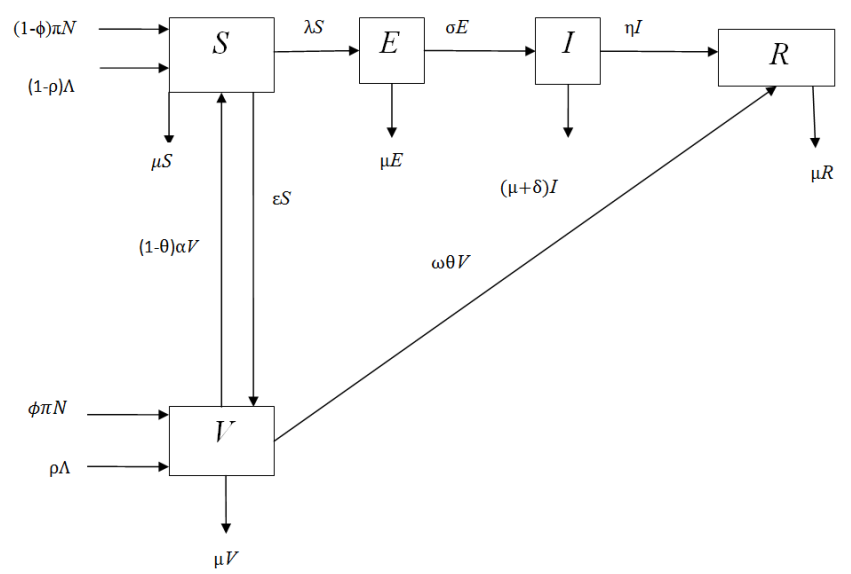

Figure 1. Compartmental Diagram for a Mathematical Model for Control and Elimination of the Transmission Dynamics of Measles.

\subsection{Differential Equations}

From the above explanation and compartmental diagram Figure 1, the transition between compartments can now be expressed by the following differential equations:

$$
\begin{gathered}
\frac{d S}{d t}=(1-\phi) \pi N+(1-\rho) \Lambda+(1-\theta) \alpha V-(\lambda+\varepsilon+\mu) S \\
\frac{d V}{d t}=\phi \pi N+\rho \Lambda+\varepsilon S-((1-\theta) \alpha+\omega \theta+\mu) V \\
\frac{d E}{d t}=\lambda S-(\sigma+\mu) E
\end{gathered}
$$

$$
\begin{aligned}
& \frac{d I}{d t}=\sigma E-(\eta+\mu+\delta) I \\
& \frac{d R}{d t}=\eta I+\omega \theta V-\mu R
\end{aligned}
$$

Where $\lambda$ is the force of infection given by;

$$
\lambda=\frac{\beta c I}{N}
$$

The total population size is:

$$
N=S+V+E+I+R
$$

Where by adding the system of equations (6-10) we get:

$$
\begin{aligned}
\frac{d N}{d t} & =\frac{d S}{d t}+\frac{d V}{d t}+\frac{d E}{d t}+\frac{d I}{d t}+\frac{d R}{d t} \\
\frac{d N}{d t} & =\Lambda+(\pi-\mu) N
\end{aligned}
$$

\subsection{Basic Properties of the Model}

\subsubsection{Dimensionless Transformation}

We use the same approach as by [26, 27, 28, 29, 30] of scaling the population of each class by the total population in order to simplify the analysis. In classes $V, S, E, I$ and $R$ we transform as follows:

$$
v=\frac{V}{N}, s=\frac{S}{N}, e=\frac{E}{N}, i=\frac{I}{N}, r=\frac{R}{N}
$$

Hence the normalized model system becomes:

$$
\begin{gathered}
\frac{d s}{d t}=(1-\phi) \pi+(1-\rho) a+(1-\theta) \alpha v-(\lambda+\varepsilon+\mu) s \\
\frac{d v}{d t}=\phi \pi+\rho a+\varepsilon s-((1-\theta) \alpha+\omega \theta+\mu) v \\
\frac{d e}{d t}=\lambda s-(\sigma+\mu) e \\
\frac{d i}{d t}=\sigma e-(\eta+\mu+\delta) i \\
\frac{d r}{d t}=\eta i+\omega \theta v-\mu r
\end{gathered}
$$

where $a=\frac{\Lambda}{N}$

Where by adding the system of equations (8-12) we get:

$$
\frac{d s}{d t}+\frac{d v}{d t}+\frac{d e}{d t}+\frac{d i}{d t}+\frac{d r}{d t}=(a+\pi)-\mu
$$

\subsubsection{Positivity of Solution}

Here we show that all state variables remain non-negative 
since they represent human population. Let $\mathrm{T}$ be a non-negative region in $\mathbb{R}^{5} ; T=\left\{(s, v, e, i, r) \in \mathbb{R}_{+}^{5} ; s(0)>\right.$ $0, i(0)>0, v(0)>0, e(0)>0, r(0)>0\}$.

We show that the solution of $\{s(t), v(t), e(t), i(t), r(t)\}$ from the system of equations (8-12) are positive for all $t \geq 0$.

For

$$
\begin{aligned}
& \frac{d s}{d t}=(1-\phi) \pi+(1-\rho) a+(1-\theta) \alpha v-(\lambda+\varepsilon+\mu) s \\
& \frac{d s}{d t} \geq(1-\phi) \pi+(1-\rho) a-(\lambda+\varepsilon+\mu) s
\end{aligned}
$$

Solving the above equation we obtain

$$
\begin{aligned}
& s(t) \geq \frac{((1-\phi) \pi+(1-\rho) a)}{(\lambda+\varepsilon+\mu)}+C \mathrm{e}^{-(\lambda+\varepsilon+\mu) t} \\
& \text { as } t \rightarrow \infty \quad s(t) \geq 0
\end{aligned}
$$

For

$$
\begin{gathered}
\frac{d v}{d t}=\phi \pi+\rho a+\varepsilon s-((1-\theta) \alpha+\omega \theta+\mu) v \\
\frac{d v}{d t} \geq \phi \pi+\rho a-((1-\theta) \alpha+\omega \theta+\mu) v
\end{gathered}
$$

Solving the above equation we obtain

$$
\begin{aligned}
& v(t) \geq \frac{(\phi \pi+\rho a)}{((1-\theta) \alpha+\omega \theta+\mu)}+C \mathrm{e}^{-[(1-\theta) \alpha+\omega \theta+\mu] t} \\
& \text { as } t \rightarrow \infty \quad v(t) \geq 0
\end{aligned}
$$

For

$$
\begin{gathered}
\frac{d e}{d t}=\lambda s-(\sigma+\mu) e \\
\frac{d e}{d t} \geq-(\sigma+\mu) e
\end{gathered}
$$

Solving the above equation we obtain

$$
\begin{aligned}
& e(t) \geq \mathrm{e}^{-C(\sigma+\mu) t} \\
& \text { as } t \rightarrow \infty \quad e(t) \geq 0
\end{aligned}
$$

For

$$
\begin{aligned}
& \frac{d i}{d t}=\sigma e-(\eta+(\mu+\delta)) i \\
& \left.\frac{d i}{d t} \geq-(\eta+\mu+\delta)\right) i
\end{aligned}
$$

Solving the above equation we obtain

$$
\begin{aligned}
& i(t) \geq \mathrm{e}^{-C[\eta+(\mu+\delta)] t} \\
& \text { as } t \rightarrow \infty \quad i(t) \geq 0
\end{aligned}
$$

For

$$
\begin{gathered}
\frac{d r}{d t}=\eta i+\omega \theta v-\mu r \\
\frac{d r}{d t} \geq-\mu r
\end{gathered}
$$

Solving the above equation we obtain

$$
\begin{aligned}
& r(t) \geq \mathrm{e}^{-C \mu} \\
& \text { as } t \rightarrow \infty \quad r(t) \geq 0
\end{aligned}
$$

\subsubsection{Invariant Region}

All state variables remain non-negative all the time because this study is based on human population. Therefore the system of equations (8-12) in the region $T$ is restricted to a non-negative condition.

where

$$
\begin{gathered}
T=\left\{(s, v, e, i, r) \in \mathbb{R}_{+}^{5} ; s>0, i>0, v>0, e>0, r>0,\right. \\
>0, s+v+e+i+r \leq 1\}
\end{gathered}
$$

The model makes biological sense where the feasible region is positively invariant.

\subsection{Model Analysis}

The system of equations (8-12) is analyzed qualitatively to give better understanding of the impact of vaccination on the control and elimination of the transmission dynamics of measles.

\subsubsection{Disease Free Equilibrium (DFE)}

The disease free equilibrium of the model system (8-12) is obtained by setting;

$$
\frac{d v}{d t}=\frac{d s}{d t}=\frac{d e}{d t}=\frac{d i}{d t}=\frac{d r}{d t}=0
$$

In case there is no disease $e=i=0$ so $s_{0}^{*}, v_{0}^{*} a n d r_{0}^{*}$ will be the proportions of susceptible, vaccinated and recovered in this case.

that is

$$
(1-\phi) \pi+(1-\rho) a+(1-\theta) \alpha v_{0}^{*}-(\varepsilon+\mu) s_{0}^{*}=0
$$

$$
\phi \pi+\rho a+\varepsilon s_{0}^{*}-[(1-\theta) \alpha+\omega \theta+\mu] v_{0}^{*}=0
$$

$$
\omega \theta v_{0}^{*}-\mu r_{0}^{*}=0
$$

and on solving equations (14-16) we get the $D F E$ of the system (8-12) which is given by:

$$
P_{0}\left(s^{*}, v^{*}, e^{*}, i^{*}, r^{*}\right)=\left(s_{0}^{*}, v_{0}^{*}, 0,0, r_{0}^{*}\right) .
$$

where $s_{0}^{*}, v_{0}^{*} r_{0}^{*}$ are given by equations (17-19).

It can also be verified that DFE is locally asymptotically stable when $R_{e}<1$. 


$$
\begin{aligned}
& s_{0}^{*}=\frac{[(1-\theta) \alpha+\omega \theta+\mu][(1-\phi) \pi+(1-\rho) a]+(1-\theta) \alpha[\phi \pi+\rho a]}{(\varepsilon+\mu)[(1-\theta) \alpha+\omega \theta+\mu]-(1-\theta) \alpha \varepsilon} \\
& v_{0}^{*}=\frac{\varepsilon[(1-\phi) \pi+(1-\rho) a]+(\lambda+\varepsilon+\mu)[\phi \pi+\rho a]}{(\varepsilon+\mu)[(1-\phi) \pi+(1-\rho) a]-(1-\theta) \varepsilon \alpha} \\
& r_{0}^{*}=\frac{\omega \theta}{\mu}\left[\frac{\varepsilon[(1-\phi) \pi+(1-\rho) a]+(\lambda+\varepsilon+\mu)[\phi \pi+\rho a]}{(\varepsilon+\mu)[(1-\phi) \pi+(1-\rho) a]-(1-\theta) \varepsilon \alpha}\right]
\end{aligned}
$$

It can be verified that $s_{0}{ }^{*}+v_{0}{ }^{*} *+r_{0}{ }^{*}=1$.

\subsubsection{The Basic Reproduction Number, $R_{0}$}

Epidemiologists have always been interested in finding the basic reproduction number of an emerging disease because this threshold parameter can tell whether a disease will die out or persist in a population. Denoted by $R_{0}$, this parameter is arguably the most important quantity in infectious disease epidemiology. It is defined as the average number of new cases (infections) produced by a single infective when introduced into a susceptible population. It is one of the first quantities estimated for emerging infectious diseases in outbreak situations [24]. It is a key epidemiological quantity, because it determines the size and duration of epidemics and is an important factor in determining targets for vaccination coverage [23]. The basic reproduction number is sought after principally because:

If $R_{0}<1$, then throughout the infectious period, each infective will produce less than one new infective on the average. This in turn implies that the disease will die out as the DFE is stable.

If $R_{0}>1$, then throughout the infectious period, each infective will produce more than one new infective on the average. This in turn implies that the disease will persist as the DFE is unstable. In other words, there will be an outbreak.

If $R_{0}$ can be determined, then the transmission parameters which will force $R_{0}$ to be less than or greater than 1 can easily be identified and control measures effectively designed.

Next, we shall find the Basic Reproduction Number of the system (8-12) using the next generation method [21].

To calculate the basic reproduction number by using a next-generation matrix, the whole population is divided into $n$ compartments in which there are $m<n$ infected compartments. In our model among five compartments we have two infected compartments

Let $x_{i}, i=1,2,3, \ldots, m$ be the numbers of infected individuals in the $i^{\text {th }}$ infected compartment at time $t . F_{i}(x)$ be the rate of appearance of new infections in compartment. $V_{i}(x)$ be the difference between rates of transfer of individuals between $i^{\text {th }}$ compartments. $V_{i}^{+}(x)$ be the rate of transfer of individuals into $i^{\text {th }}$ compartment by all other means. $V_{i}^{-}(x)$ be the rate of transfer of individuals out of $i^{t h}$ compartment.

$$
\frac{d x_{i}}{d t}=F_{i}(x)-V_{i}(x), \text { where } V_{i}(x)=\left[V_{i}^{-}(x)-V_{i}^{+}(x)\right]
$$

The above equation can also be written as:

$$
\frac{d x_{i}}{d t}=F(x)-V(x)
$$

where $F(x)=\left(F_{1}(x), F_{2}(x), \ldots \ldots, F_{m}(x)\right)^{T}$,

$$
V(x)=\left(V_{1}(x), V_{2}(x), \ldots \ldots, V_{m}(x)\right)^{T}
$$

From equations (10) and (11);

$$
\begin{gathered}
\frac{d e}{d t}=\lambda s-(\sigma+\mu) e \\
\frac{d i}{d t}=\sigma e-[\eta+(\mu+\delta)] i \\
\lambda=\beta i c
\end{gathered}
$$

we can say from our explanation above that $x_{1}=e$ and $x_{2}=i$

$$
\begin{gathered}
F=\left(F_{1}, F_{2}\right)^{T} \\
F=(\beta i c s, 0)^{T} \\
V=\left(V_{1}, V_{2}\right)^{T} \\
V=[(\sigma+\mu) e,-\sigma e+(\eta+\mu+\delta) i]^{T}
\end{gathered}
$$

So we define $F_{i}$ and $V_{i}$ as:

$$
F_{i}=\left[\begin{array}{c}
\beta i c s \\
0
\end{array}\right] \quad V_{i}=\left[\begin{array}{c}
(\sigma+\mu) e \\
-\sigma e+(\eta+\mu+\delta) i
\end{array}\right]
$$

Let $x_{0}$ be the disease-free equilibrium. The values of the Jacobian matrices $F(x)$ and $V(x)$ are:

We differentiate $F_{i}$ with respect to $e$ and $i$ and get $F$ :

$$
\begin{aligned}
F & =D F\left(x_{0}\right)=\frac{\partial F_{i}\left(x_{0}\right)}{\partial x_{j}} \\
F & =\left[\begin{array}{ll}
\frac{\partial F_{1}}{\partial e} & \frac{\partial F_{1}}{\partial i} \\
\frac{\partial F_{2}}{\partial e} & \frac{\partial F_{2}}{\partial i}
\end{array}\right]=\left[\begin{array}{cc}
0 & \beta c s_{0}^{*} \\
0 & 0
\end{array}\right]
\end{aligned}
$$

We differentiate $V_{i}$ with respect to $e$ and $i$ and get $V$ : 


$$
\begin{aligned}
V & =D V\left(x_{0}\right)=\frac{\partial V_{i}\left(x_{0}\right)}{\partial x_{j}} \\
V & =\left[\begin{array}{ll}
\frac{\partial V_{1}}{\partial e} & \frac{\partial V_{1}}{\partial i} \\
\frac{\partial V_{2}}{\partial e} & \frac{\partial V_{2}}{\partial i}
\end{array}\right]=\left[\begin{array}{cc}
(\sigma+\mu) & 0 \\
-\sigma & (\eta+\mu+\delta)
\end{array}\right]
\end{aligned}
$$

We find the inverse of $V$ and get:

$$
V^{-1}=\left[\begin{array}{cc}
\frac{1}{(\sigma+\mu)} & 0 \\
\frac{\sigma}{(\sigma+\mu)(\eta+\mu+\delta)} & \frac{1}{(\eta+\mu+\delta)}
\end{array}\right]
$$

Now, the matrix $F V^{-1}$ is known as the next-generation matrix. The largest eigenvalue or spectral radius $F V^{-1}$ of is the effective reproduction number of the model.

$$
\begin{aligned}
& F V^{-1}=\left[\frac{\partial F_{i}\left(x_{0}\right)}{\partial x_{j}}\right]\left[\frac{\partial V_{i}\left(x_{0}\right)}{\partial x_{j}}\right]^{-1} \\
& F V^{-1}=\left[\begin{array}{ccc}
0 & \beta c s_{0}^{*} \\
0 & 0
\end{array}\right]\left[\begin{array}{cc}
\frac{1}{(\sigma+\mu)} & 0 \\
\frac{\sigma}{(\sigma+\mu)(\eta+\mu+\delta)} & \frac{1}{(\eta+\mu+\delta)}
\end{array}\right] \\
& F V^{-1}=\left[\begin{array}{cc}
\beta c s_{0}^{*} \sigma & \beta c s_{0}^{*} \\
\frac{(\eta+\mu+\delta)}{(\sigma+\mu)(\eta+\mu+\delta)} & \frac{0}{(\eta+\mu+}
\end{array}\right]
\end{aligned}
$$

The eigenvalues, $\lambda$ of equation (20) can be computed from the characteristic equation:

$$
\begin{gathered}
\left|F V^{-1}-\lambda I\right|=0 . \\
\quad\left|\begin{array}{cc}
\frac{\beta c s_{0}^{*} \sigma}{(\sigma+\mu)(\eta+\mu+\delta)}-\lambda & \frac{\beta c s_{0}^{*}}{(\eta+\mu+\delta)} \\
0 & -\lambda
\end{array}\right|=0 \\
\lambda\left(\frac{\beta c s_{0}^{*} \sigma}{(\sigma+\mu)(\eta+\mu+\delta)}-\lambda\right)=0 \\
\lambda_{1}=0 \quad \text { and } \quad \lambda_{2}=\frac{\beta c s_{0}^{*} \sigma}{(\sigma+\mu)(\eta+\mu+\delta)}
\end{gathered}
$$

The largest eigenvalue is obviously $\lambda_{2}$ and it becomes equal to the effective reproduction number of the model. If we substitute $S_{0}^{*}$ from equation (17) we get the effective reproduction number denoted by $R_{e}$ (here all control strategies have been considered) equation (21) below:

$R_{e}=\frac{\beta c \sigma\{[(1-\theta) \alpha+\omega \theta+\mu][(1-\phi) \pi+(1-\rho) a]+(1-\theta) \alpha[\phi \pi+\rho a]\}}{(\sigma+\mu)(\eta+\mu+\delta)\{(\varepsilon+\mu)[(1-\theta) \alpha+\omega \theta+\mu]-(1-\theta) \alpha \varepsilon\}}$
When there is no any control strategy, then $\varepsilon=\omega=\phi=$ $\rho=0$ hence $\theta=0, \alpha=0$ so we get the basic reproduction number denoted by $R_{0}$ given by equation (22) below:

$$
R_{0}=\frac{\beta c \sigma(\pi+a)}{\mu(\sigma+\mu)(\eta+\delta+\mu)}
$$

When vaccination is administered to newborns and immigrants only, leaving away Susceptibles individuals, We set $\mathcal{\varepsilon}=\alpha=0$ and obtain the reproduction number denoted by $R e_{1}$ given by the equation (23) below:

$$
R_{e 1}=\frac{\beta c \sigma\{[\omega \theta+\mu][(1-\phi) \pi+(1-\rho) a]\}}{\mu(\sigma+\mu)(\eta+\mu+\delta)(\omega \theta+\mu)}
$$

\section{Simulation and Discussion}

In this section we employ MATLAB to simulate a mathematical model formulated, provide sensitivity analysis and discuss the results.

\subsection{Simulation and Discussion}

A mathematical model for control and elimination of the transmission dynamics of measles is formulated and analyzed. The main objective of this study was to assess the impact of immunization strategies on the transmission dynamics of the disease. In order to support the analytical results, graphical representations showing the variations in reproduction numbers with respect to exposure rate are provided in Figure 2 as well and time graphs of different state variables. Since, most of the parameters were not readily available; therefore we estimated them and obtain the rest from other sources just for the purpose of illustration. Table 3 shows the set of parameter values which were used.

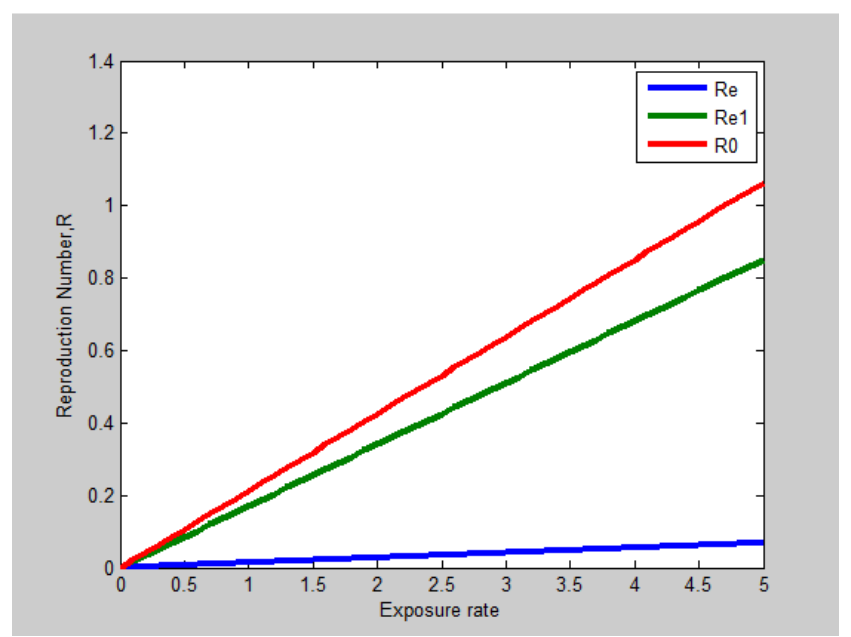

Figure 2. Variations in reproduction number with respect to exposure rate. 
Table 3. Value of parameters used in the model.

\begin{tabular}{llll}
\hline Parameter & Description & Value/range & Source \\
\hline$\pi$ & Per Capita birth rate & 0.02755 per year & {$[25]$} \\
$\phi$ & Proportions of newborns who are vaccinated & 0.5 also varies with scenario $(0.0-1.0)$ & {$[26,27]$} \\
$\rho$ & Proportions of immigrants who are vaccinated & 0.7 also varies with scenario $(0.0-1.0)$ & {$[26,27]$} \\
$a$ & Arrival rate & 0.02755 per year & 0.09091 per year \\
$c$ & Per Capita contact rate & 0.125 per year & {$[25]$} \\
$\delta$ & Death due to disease & 0.00875 per year & {$[17]$} \\
$\mu$ & Per Capita natural mortality rate & varies with scenario $(0.08-0.7)$ & {$[25]$} \\
$\beta$ & Probability of one infected individual to become infectious & 0.096 per year & {$[18]$} \\
$\lambda$ & Force of infection, $\lambda=\frac{\beta c I}{N}$ & 0.125 per year & {$[26,27]$} \\
$\sigma$ & Progression rate from latent to infectious & 0.14286 per year varies with scenario $(0.0-1.0)$ & {$[25]$} \\
$\eta$ & Recovery rate of treated infectious individuals & 0.167 per year & Estimated \\
$\alpha$ & The rate of waning of first dose of vaccine & 0.8 per year & {$[26,27]$} \\
$\omega$ & The rate of receiving second dose of vaccine & 0.7 per year varies with scenario $(0.0-1.0)$ & {$[26,27]$} \\
$\varepsilon$ & Proportion of individuals who received a first vaccination & 0.5 varies with scenario $(0.0-1.0)$ & {$[26,27]$} \\
$\theta$ & Proportion of individuals who are vaccinated twice & & \\
\hline
\end{tabular}

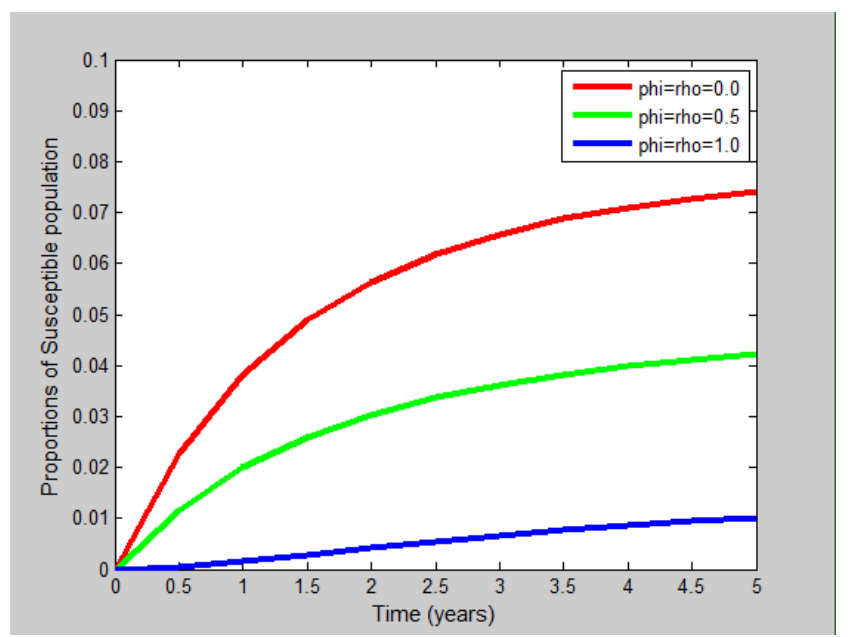

Figure 3. Susceptible population in an outbreak, varying the proportion of newborns and immigrants vaccinated (phi=rho=0.0, 0.5, 1.0).

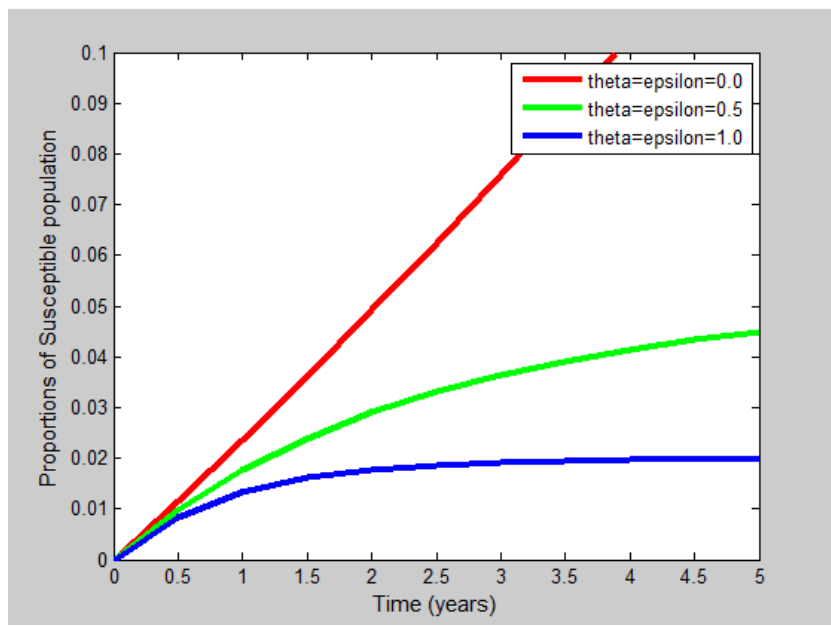

Figure 4. Susceptible population in an outbreak, varying the proportion of first and second vaccination (theta $=$ epsilon $=0.0,0.5,1.0$ ).

Figure 2 shows that $R_{e}<R_{e 1}<R_{0}$,we see from the figure 2 that $R_{0}$ is worst case scenario, it occurs when there is no vaccination strategy to control the epidemic, here an individual recovers naturally. The basic reproduction number $R_{0}$ is at the peak, this implies that there is a high increase in reproduction number with respect to exposure rate. Such increase results in the outbreak of measles in the community.

The middle graph $R_{e_{1}}$ from the same figure 2, shows effects of vaccinating immigrants and newborns only leaving away the susceptible population, it can be noted that even though just a proportion of the population was vaccinated but still have a significant contribution on diminishing the disease as compared to when no any control is in place $\left(R_{0}\right)$.

The best case scenario occurs at graph $R_{e}$ of the similar figure 2 , here vaccination is offered to newborns, immigrants and the susceptible adults in two doses, we note that $R_{e}$ has the least value of increase in reproduction number with respect to exposure rate, which implies that measles can be eradicated from the community if two dose vaccination policies is seriously targeted to a large population.

Figure 3 shows that the increase in vaccination coverage to both the newborns and immigrants causes a reduction in the susceptible population and hence reducing the risk of an outbreak.

It can be realized from figure 4 that when vaccination programmes are effectively implemented to the population, it may reach a stage in which the disease fail to erupt since there are very few susceptible individuals to infect, such a phenomenon is known as herd immunity.

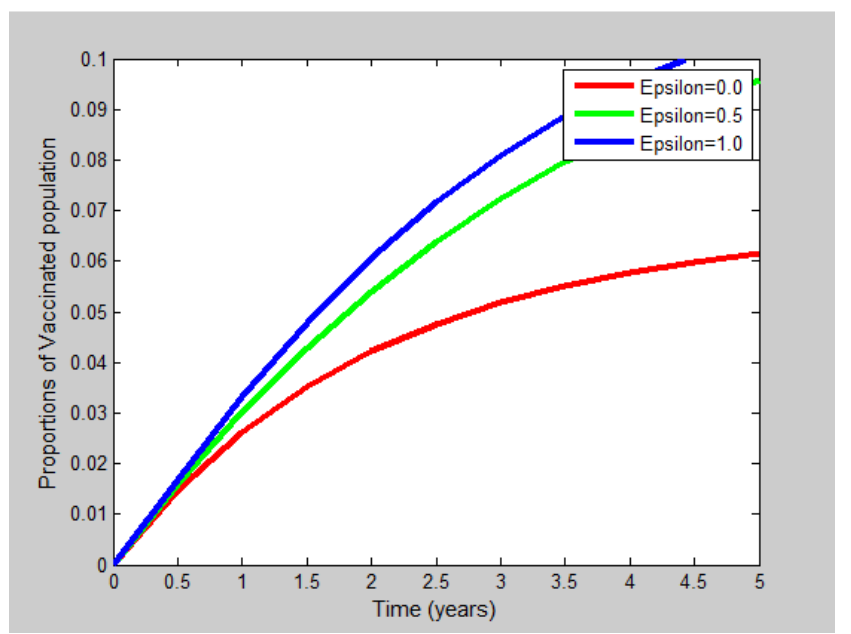

Figure 5. Vaccinated population in an outbreak, varying the proportion of first dose vaccination (epsilon $=0.0,0.5,1.0$ ).

The above figure 5 shows the number of vaccinated 
individuals increase by offering first dose of vaccine to susceptible individuals in the population and therefore reducing the number of susceptible adults and children in the population.

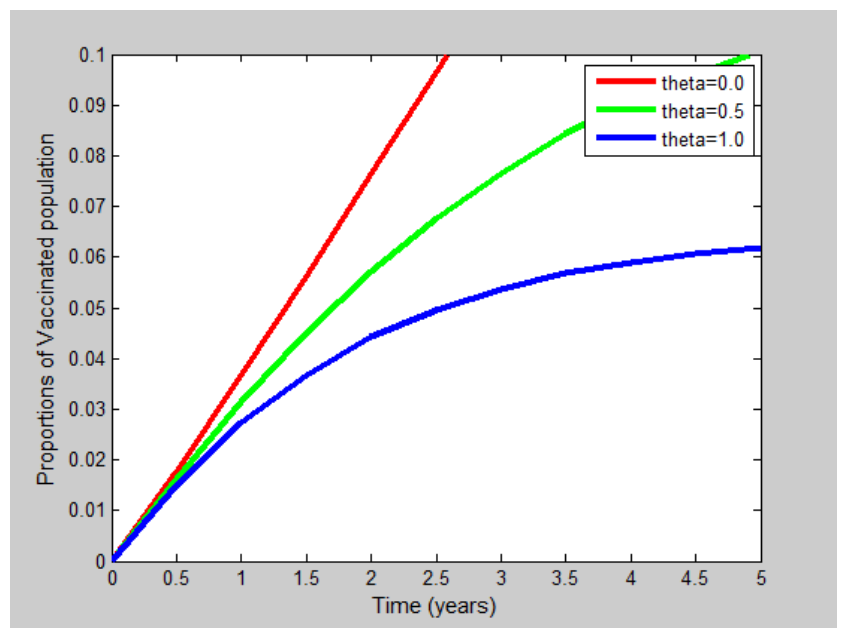

Figure 6. Vaccinated population in an outbreak, varying the proportion of second vaccination (theta $=0.0,0.5,1.0$ ).

Figure 6 above shows that provision of second dose of vaccine increase the number of individuals who cannot be infected with the disease by reducing the number of those who just received first dose of vaccine.

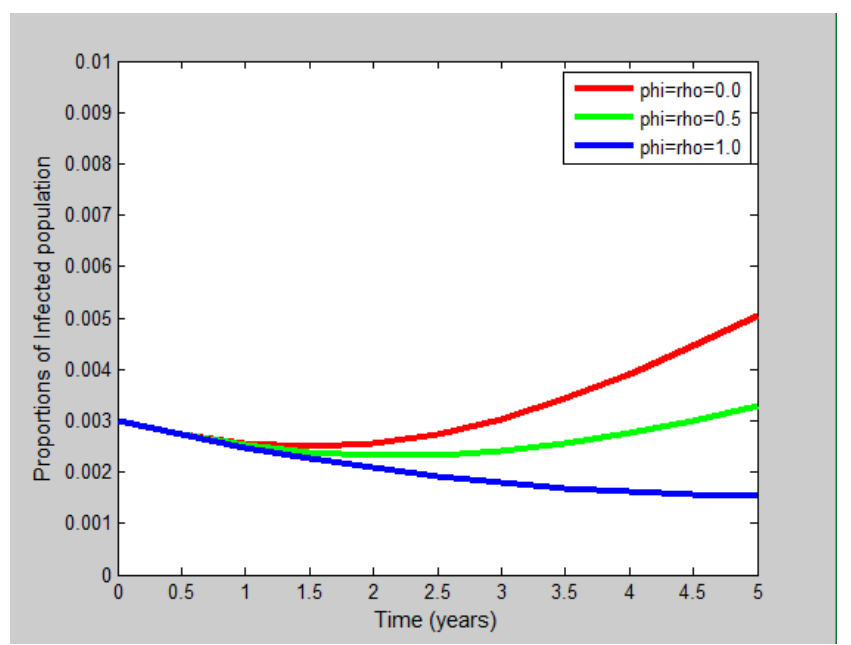

Figure 7. Infected population in an outbreak, varying the proportion of newborns and immigrants vaccinated (phi $=r h o=0.0,0.5,1.0$ ).

We can observe from figure 7 above that if more newborns and immigrants receive vaccination then the likelihood of individuals to be infected with the disease becomes very small. This in turn can lead to the disease to die out in a population.

It can be seen from Figure 8 that the proportion of infected individuals decrease with an increase in vaccination coverage of both dose 1 and dose 2.This is also attributed by the fact that less people will be susceptible as they will be immune to the disease.

We can observe from Figure 9 how the provision of fist dose and second dose of vaccination increase the number individual who are immune to the disease and therefore reducing the risk of an outbreak in the population.

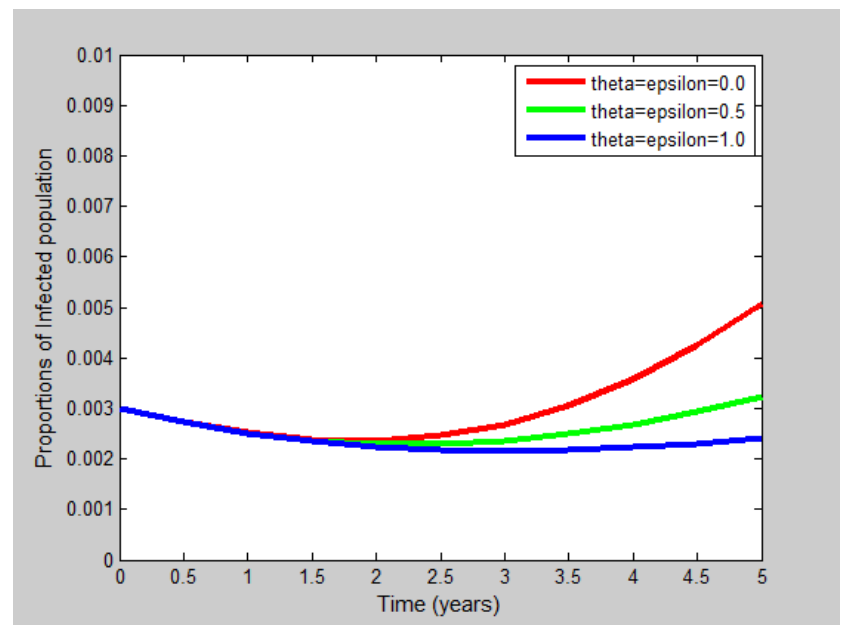

Figure 8. Infected population in an outbreak, varying the proportion of first and second dose vaccinated (theta $=$ epsilon $=0.0,0.5,1.0$ ).

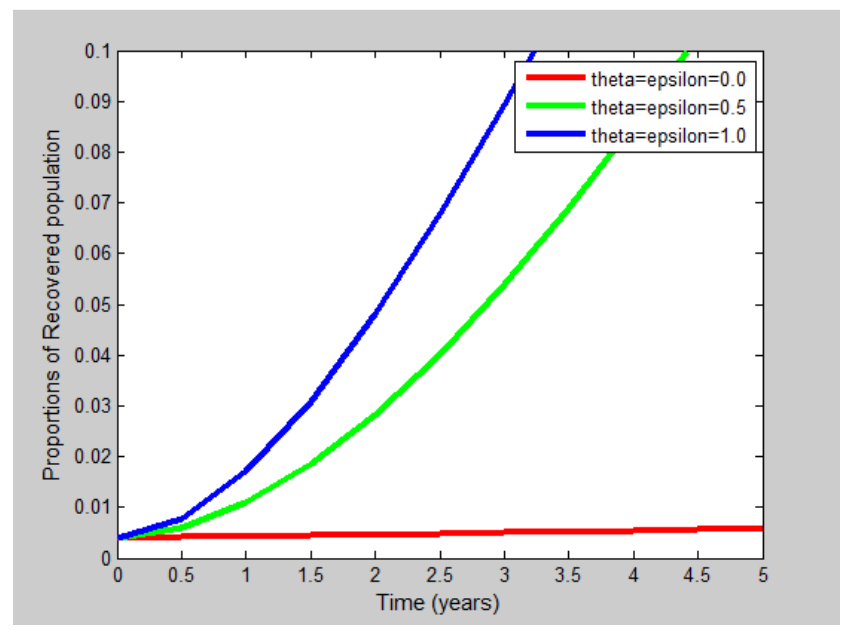

Figure 9. Recovered population in an outbreak, varying the proportion of first and second dose vaccinated (theta $=$ epsilon $=0.0,0.5,1.0$ ).

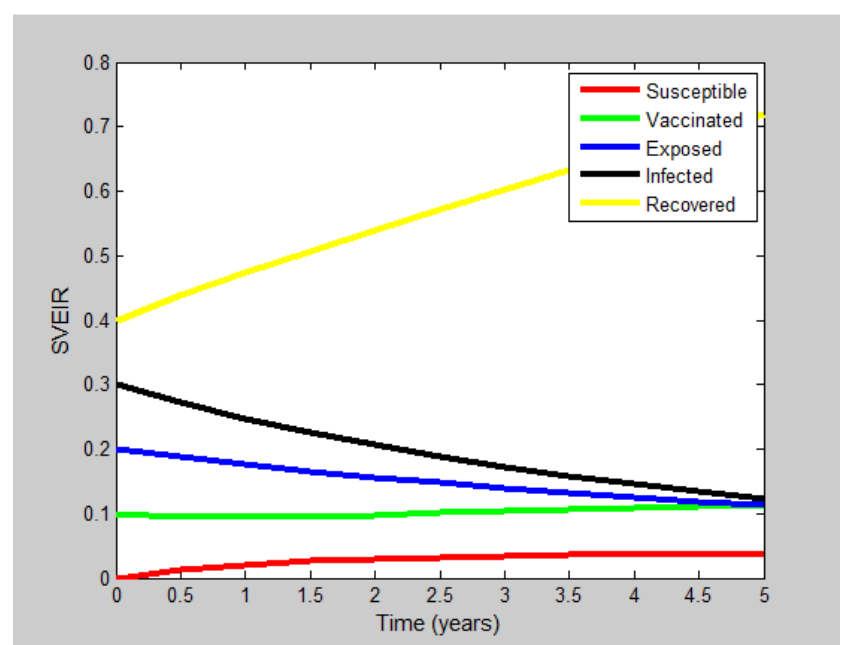

Figure 10. Combined Susceptible, Vaccinated, Exposed, Infectious and Recovered population.

Figure 10 shows that the model provides the illustration for control and elimination of the transmission dynamics of 
measles. We can observe that recovered individuals can be increased by increasing provision of vaccination and consequently reducing the susceptible and infectious individuals.

\subsection{Sensitivity Analysis}

Sensitivity analysis is used to determine how "sensitive" a model is to changes in the value of the parameters of the model and to changes in the structure of the model. Sensitivity analysis helps to build confidence in the model by studying the uncertainties that are often associated with parameters in models. Sensitivity indices allow us to measure the relative change in a state variable when a parameter changes. Sensitivity analysis is commonly used to determine the robustness of model predictions to parameter values (since there are usually errors in data collection and presumed parameter values). Thus we use it to discover parameters that have a high impact on $R_{0}$ and should be targeted by intervention strategies. If the result is negative, then the relationship between the parameters and $R_{0}$ is inversely proportional. In this case, we will take the modulus of the sensitivity index so that we can deduce the size of the effect of changing that parameter. On the other hand, a positive sensitivity index means an increase in the value of a parameter $[26,28,30]$. The explicit expression of $R_{0}$ is given by the equation (22). Since $R_{0}$ depends only on six parameters, we derive an analytical expression for its sensitivity to each parameter using the normalized forward sensitivity index as by [31] as follows:

$$
\begin{aligned}
& \Upsilon_{c}^{R_{0}}=\frac{\partial R_{0}}{\partial c} \times \frac{c}{R_{0}}=+1 \\
& \Upsilon_{\beta}^{R_{0}}=\frac{\partial R_{0}}{\partial \beta} \times \frac{\beta}{R_{0}}=+1 \\
& \Upsilon_{\delta}^{R_{0}}=\frac{\partial R_{0}}{\partial \delta} \times \frac{\delta}{R_{0}}=-0.4518998
\end{aligned}
$$

The rest of sensitivity indices for all parameters used in equation (22) can be computed in the similar approach. Table below shows the sensitivity indices of $R_{0}$ with respect to the eight parameters.

Table 4. Sensitivity Indices.

\begin{tabular}{lll}
\hline Parameter & Description & $\begin{array}{l}\text { Sensitivity } \\
\text { Index }\end{array}$ \\
\hline$\beta$ & Probability of one infected individual to & +1 \\
$c$ & become infectious. & +1 \\
$\sigma$ & Per capita contact rate. & 0.0654 \\
$\pi$ & Progression rate from latent to infectious. & 0.5 \\
$a$ & Per capita birth rate. & 0.5 \\
$\mu$ & Arrival rate. & -1.0971 \\
$\delta$ & Per capita natural mortality rate. & -0.4519 \\
$\eta$ & Death due to disease & -0.5165 \\
\hline
\end{tabular}

From Table above, we can obtain $\Upsilon_{\beta}^{R_{0}}=\Upsilon_{c}^{R_{0}}=+1$, this means that an increase in $c$ or $\beta$ will cause an increase of exactly the same proportion in $\mathrm{R}_{0}$. Similarly, a decrease in $c$ or $\beta$ will causes a decrease in $R_{0}$, as they are directly proportional. We can also note that $\mu, \delta, \eta<0$ hence these parameters are inversely proportional to $R_{0}$.

It can be seen that, the most sensitive parameters are $c$ and $\beta$ followed by $a$ then $\pi$ then $\sigma$, then $\delta$, then $\eta$ and the least sensitive parameter is $\mu$.

Therefore, to minimize measles transmission in a population, this study recommends that, vaccination should be implemented. This is due to the fact that, vaccination reduces the likelihood of an individual to be infected, also treatment of latently infected people reduces the progression rate to infectious stage and treatment of infectious people will stop them from transmitting the disease.

\section{Conclusions, Recommendations and Future Work}

\subsection{Conclusion}

The model has shown importance of measles vaccination in preventing transmission within a population. The model strongly indicated that the spread of a disease largely depend on the contact rates with infected individuals within a population.

It is also realized that if the proportion of the population that is immune exceeds the herd immunity level of measles, then the disease can no longer persist in the population. In fact, this level can be attained by mass vaccination.

\subsection{Recommendations}

Eradication of contagious diseases such as measles has remained one of the biggest challenge facing developing countries. It is realized that the herd immunity level for the disease is high, and mostly when there is an outbreak of the disease, and there is an introduction of a mass vaccination programme which can cover large number of the population, not everybody will be immune because vaccine efficacy is usually not $100 \%$. It therefore means that part of the population will be immune and others will be vaccinated but not immune [1]. Therefore there is an urgent need for any country to come up with some new control strategies and more efficient ones to fight the spread of the disease in the country.

From the results of this project the following control strategies are recommended:

i. Since the model shows that the spread of the disease largely depend on the contact rate, therefore effort should be made to minimize unnecessary contact with measles infected individuals, this will reduce risk of an outbreak.

ii. To attain high level herd immunity for the disease, mass vaccination exercise should be encouraged to cover the majority of the population to prevent outbreak of the disease in developing country.

iii. Measles infected individuals should be treated early this will limit its transmission. 


\subsection{Future Work}

Based on the model of this study, it is proposed that future work should consider the following:

i. Any researcher may use this model as a foundation to perform a case study at a specific region and obtain practical results.

ii. Carrying out cost-effectiveness analysis of the measles immunization model.

\section{References}

[1] A. Mazer, Sankale, Guide de medicine en Afrique et Ocean Indien, EDICEF, Paris (1988).

[2] M. B. A. Oldstone, Viruses, Plagues, and History, Oxford University Press, New York, USA (1998), 73-89.

[3] Department of Health Statistics and Informatics. WHO mortality Database .Geneva: World Health Organization, 2011.

[4] WHO vaccine-preventable diseases: monitoring system. Geneva: World Health Organization, 2010.

[5] Burton A, Gacic-Dobo M, Karimov R, Kowalski R. A computational logic-based representation of the WHO and UNICEF estimates of national immunization coverage. http://www.doc.ic.ac.uk/ rak/papers/wuenic.pdf (accessed April 14, 2012).

[6] Harpaz R. Completeness of measles case reporting: review of estimates for the United States. J Infect Dis 2004; 189 (suppl 1): S185-90.

[7] Catherine Comiskey B A (Mod). (1988) A mathematical Model for Measles Epidemics in Ireland. Msc Thesis. National Institute of Higher Education.

[8] Ministry of Health, Manatu Hauora. (1998) Predicting and Preventing Measles Epidemics in New Zealand: Application of Mathematical Model.

[9] M. G. Roberts and M. I. Tobias (2000). Predicting and Preventing measles epidemics in New Zealand: Application of Mathematical Model. Cambridge University Press, Vol 124,pp. 279-287.

[10] C. R. Mac Intyre, N. J. Gay, H. F. Gidding, B. P. Hull, G. L. Gilbert and P. B. McIntyre (2002). A Mathematical model to the impact of the measles control campaign on the potential for measles transmission in Australia. International Journal of Infectious Diseases, Vol 6,pp. 277-282.

[11] J. Mossong and C. P. Muller. (2003) Modeling measles re-emergence as a result of waning of immunity in vaccinated populations. Science direct, Vaccine 21, pp. 4597-4603.

[12] O. M. Tessa. (2006) Mathematical Model for control of measles by vaccination. Abdou Moumouni University, Niamey, Niger, pp. 31-36.

[13] E. A. Bakare, Y. A. Adekunle and K. O. Kadiri. (2012) Modelling and Simulation of the Dynamics of the Transmission of Measles. International Jounal of Computer Trends and Technology, Vol 3, pp. 174-178.

[14] S. O. Siabouh and I. A. Adetunde. (2013) Mathematical Model for the study of measles in Cape Coast Metropolis. International Journal of Mordern Biology and Medicine, Vol 4(2), pp. 110-133.

[15] A. A. Momoh, M. O. Ibrahim, I. J. Uwanta, S. B. Manga. (2013) Mathematical Model for control of Measles Epidemiology. International Journal of Pure and Applied Mathematics, Vol 67, pp. 707-718.

[16] A. A. Momoh, M. O. Ibrahim, I. J. Uwanta, S. B. Manga. (2013) Modelling the effects of vaccination on the transmission dynamics of measles. International Journal of Pure and Applied Mathematics, Vol 88, pp. 381-390.

[17] M. O. Fred, J. K. Sigey, J. A. Okello, J. M. Okwyo and G. J. Kang' ethe. (2014) Mathematical Modelling on the Control of Measles by Vaccination: Case Study of KISII Country, Kenya. The SIJ Transactions on Computer Science Engineering and Its Applications (CSEA), Vol 2,pp. 61-69.

[18] J. M. Ochoche and R. I. Gweryina. (2014) A Mathematical Model of Measles with Vaccination and Two Phases of Infectiousness. IOSR Jounal of Mathematics, Vol 10, pp. 95-105.

[19] G. Bolarian. (2014) On the Dynamical Analysis of a new Model for Measles Infection. International Journal of Mathematics Trends and Technology, Vol 7, pp. 144-155.

[20] Verguet S, et al. Controlling measles using supplemental immunization activities: A mathematical model to inform optimal policy. Vaccine (2015), http://dx.doi.org/10.1016/j.vaccine.2014.11.050.

[21] Van Den Driessche, P and Watmough, J (2002). Reproduction numbers and Sub-threshold endemic equilibria for compartmental models of disease transmission. Mathematical Biosciences, Vol 180, pp. 29-48.

[22] Schenzle D. (1984). An age-structured model of pre- and post-vaccination measles transmission. Math Med Biol Vol 1(2), pp.169-191.

[23] R. F. Grais, M. J. Ferrari, C. Dubray, O. N. Bjørnstad, B. T. Grenfell, A. Djibo, F. Fermon, P. J. Guerin.(2006) Estimating transmission intensity for a measles epidemic in Niamey, Niger: lessons for intervention. Royal society of tropical medicine and hygiene,Vol100,pp. $867-873$.

[24] O. Diekmann, J. A. P. Heesterbeek and M. G. Roberts. (2009) The construction of next-generation matrices for compartmental epidemic models. J. R. Soc. Interface, (doi:10.1098/rsif.2009.0386).

[25] Anes Tawhir. (2012). Modelling and Control of Measles Transmission in Ghana. Master of Philosophy thesis. Kwame Nkrumah University of Science and Technology.

[26] Stephen, E., Dmitry K and Silas M.(2014). Modeling and Stability Analysis for a Varicella Zoster Virus Model with Vaccination. Applied and Computational Mathematics. Vol. 3, No. 4, pp. 150-162.doi: 10.11648/j.acm.20140304.16.

[27] Stephen, E., Dmitry K and Silas M.(2014). Modeling the Impact of Immunization on the epidemiology of Varicella Zoster Virus. Mathematical theory and Modeling.Vol.4, No. 8, pp.46-56.

[28] Stephen, E. and N. Nyerere. (2015). A Mathematical Model for the Dynamics of Cholera with Control Measures. Applied and Computational Mathematics. Vol.4, No.2, pp.53-63. doi: 10.11648/j.acm.20150402.14. 
[29] N. Nyerere, L. S. Luboobi and Y. Nkansah-Gyekye. Modeling the Effect of Screening and Treatment on the Transmission of Tuberculosis Infections. Mathematical theory and Modeling. Vol.4, No. 7, 2014, pp.51-62.

[30] N. Nyerere, L. S. Luboobi and Y. Nkansah-Gyekye. Bifurcation and Stability analysis of the dynamics of Tuberculosis model incorporating, vaccination, Screening and treatment, Communications in Mathematical biology and Neuroscience, Vol 2014(2014), Article ID 3.

[31] Chitnis, N., Hyman, J. M., and Cusching, J. M. (2008). Determining important Parameters in the spread of malaria through the sensitivity analysis of a mathematical Model. Bulletin of Mathematical Biology 70(5):1272-12. 